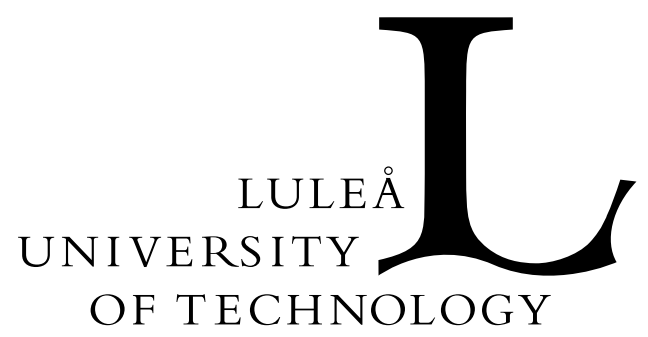

Division of Signal Processing

Visiting address: Universitetsområdet, Porsön, Luleå Postal address: SE-971 87, Luleå, Sweden

Telephone: +46920910 00. Fax: +4692072043

URL: http://www.sm.luth.se/csee/sp/

\title{
Time and Frequency Offset in OFDM Systems Employing Pulse Shaping
}

\section{Daniel Landström, Julia Martinez Arenas, Jan-Jaap van de Beek, Per Ola Börjesson, Marie-Laure Boucheret, Per Ödling}

In Proceedings of IEEE International Conference on Universal Personal Communication (ICUCP '97), pp. 279-283, San Diego, California, USA, October 1997.

(C) 1997 IEEE. Personal use of this material is permitted. However, permission to reprint/republish this material for advertising or promotional purposes or for creating new collective works for resale or redistribution to servers or lists, or to reuse any copyrighted component of this work in other works must be obtained from the IEEE. 


\title{
Time and Frequency Offset Estimation in OFDM Systems Employing Pulse Shaping*
}

\author{
Daniel Landström ${ }^{1} \quad$ Julia Martinez Arenas ${ }^{2} \quad$ Jan-Jaap van de Beek $^{1}$ \\ Per Ola Börjesson ${ }^{1} \quad$ Marie-Laure Boucheret ${ }^{2} \quad$ Per Ödling $^{1}$ \\ ${ }^{1}$ Luleå University, Div. of Signal Processing and the \\ Centre for Distance-Spanning Technology, S-971 87 Luleå, Sweden \\ ${ }^{2}$ Ecole National Supérieure de Télécommunications, \\ Site de Toulouse, BP4004 CEDEX, Toulouse, France
}

\begin{abstract}
Time and frequency offset estimation is addressed in an OFDM system that uses a cyclic prefix and pulse shaping. Earlier work has presented a method that avoids the use of pilots by exploiting the inherent correlation in the cyclically extended OFDM symbol. In this paper this technique is extended to include systems with pulse shaping. The joint Maximum Likelihood estimator of time and frequency offsets for such systems is presented and evaluated. An implementable structure of the extended estimator is given. Simulations show that it is possible to maintain the performance when introducing pulse shaping into an OFDM system.
\end{abstract}

\section{Introduction}

In this paper we consider a mobile communication system based on Orthogonal Frequency Division Multiplexing (OFDM) [1]. Time and frequency offset estimation are important topics in the design of OFDM systems, and often give rise to challenging problems [2]-[4]. In, for example, [5]-[9] estimators are presented that operate without the use of pilots. In particular the joint Maximum Likelihood (ML) estimator for time and frequency offsets in OFDM systems without pulse shaping was presented in [9].

Because pulse shaping has been proposed in OFDM systems for improving sidelobe suppression [10] in this paper, we extend previous work on time and frequency offset estimation to systems with pulse shaping.

The system under consideration is a time-divided multiuser system for mobile communication. In this system the users transmit in different time slots using all subcarriers. The considered system also employs pulse shaping. The aim of this paper is to present a structure for an estimator of time and frequency offset, that supports the synchronization of a mobile to the base station. We extend the signal model in [9] to a more general model that covers arbitrary

\footnotetext{
*This work has been supported by Telia Research AB under NUTEK contract.
}

pulse shapes and interference from neighbouring time slots. From this model we derive the joint Maximum Likelihood estimator of the time and frequency offsets. For the derivation of the estimator we assume an AWGN channel, i.e., that the received OFDM symbols are disturbed by additive white Gaussian noise only. The derivation of the estimator is based on a single received symbol. We then show the performance of the estimator for time-dispersive channels in mobile environments through simulations.

The paper is organized as follows. Section 2 gives a mathematical model of the estimation problem. The associated ML estimator of time and carrier frequency offsets and an implementation structure are presented in Section 3. In Section 4 we will give interpretations and choices for the power profile of the received signal. Simulated performance results for the estimator are presented in Section 5. Finally, we conclude the paper in Section 6 .

\section{Signal model}

The purpose of the model is to estimate time and frequency offset. The OFDM symbol is corrupted by white Gaussian noise and may be preceded and followed by other OFDM symbols. We model our symbol and its neighbour symbols in adjacent time slots by means of an expected power profile. This function depends on the pulse shape employed in the system, the probability that an adjacent time slot is being used, jitter in the arrival times of the adjacent pulses, etc. In this and the following section we present a mathematical model and derive the estimator for an arbitrary choice of the power profile. Interpretations and examples of particular profiles are developed in Section 4.

In an OFDM system with a reasonably large number of tones, the sampled baseband time-domain OFDM signal has statistical properties similar to a discrete-time Gaussian process [11]. In our model we assume that the transmitted signal $s(k)$ is a Gaussian process ${ }^{1}$ with variance $\sigma_{s}^{2}$. Let $L$ samples of the $\mathrm{N}$-sample signal $s(k)$ be repeated as the

\footnotetext{
${ }^{1}$ The Gaussian assumption determines the optimality for the estimator derived in Section 3.
} 
cyclic prefix, i.e., $s(k)=s(k+N), k \in[0, L-1]$ and $L<N$. The length of one OFDM symbol is thus $N+L$ samples of which $L$ samples constitute the cyclic prefix. Our estimator will be derived assuming an infinite observation interval.

We model the received signal $r(k)$ as

$$
r(k)=g(k-\theta) s(k-\theta) e^{j 2 \pi \varepsilon k / N}+n(k) .
$$

The attenuation $g(k)$ models the power profile and the parameters $\theta$ and $\varepsilon$ represent the time and the carrier frequency offsets respectively. The function $n(k)$ is additive complex white Gaussian noise at the receiver.

We assume that the adjacent OFDM symbols' may come from other users. Hence they have different timing offsets, and thus we make no use of the redundant information in their cyclic prefixes. Time and frequency offsets will be estimated based on one symbol, as if they were statistically independent in time. This method could be extended with averaging of the likelihood function or filtering the estimates if correlation between symbols exists.

\section{Estimator of time and frequency offset}

In order to derive the optimal joint ML estimator of the time offset $\theta$ and the carrier frequency offset $\varepsilon$, we investigate the $\log$-likelihood function of the received signal vector $\mathbf{r} \triangleq$ $[r(1), r(2), \ldots, r(\infty)]$. We follow the same procedure as in [9]. The log-likelihood function can be expressed as the logarithm of the conditional probability density function of the observed vector $\mathbf{r}$, as in

$$
\Lambda(\theta, \varepsilon)=\log f(\mathbf{r} \mid \theta, \varepsilon) .
$$

The samples in $\mathbf{r}$ are mutually uncorrelated except for those samples that are repeated in the cyclic prefix. The joint probability density function for $(r(k), r(k+N)), k \in$ $[\theta, \theta+L-1]$ (samples coupled through the cyclic extension) is denoted by $f(r(k), r(k+N))$ while $f(r(k))$ denotes the density function for other values of $k$. Notice that $f(\cdot)$ is used both for one- and two-dimensional distributions.

Using the correlation due to the cyclic prefix in the received signal, the log-likelihood function becomes

$$
\Lambda(\theta, \varepsilon)=\log \left(\prod_{k \in I} f(r(k), r(k+N)) \prod_{k \notin I \cup I^{\prime}} f(r(k))\right)
$$

where $I=[\theta, \theta+L-1]$ and $I^{\prime}=[\theta+N, \theta+N+L-1]$ are the intervals coupled through the cyclic prefix. A more convenient form for the log-likelihood function is

$\Lambda(\theta, \varepsilon)=\sum_{k=\theta}^{\theta+L-1} \log \frac{f(r(k), r(k+N))}{f(r(k)) f(r(k+N))}+\sum_{k=-\infty}^{\infty} \log f(r(k))$ where the first sum stems from the cyclic prefix and the second primarily considers the power profile of the received symbol. Notice that, in this case as opposed to the case in [9], the second sum is dependent on the timing offset $\theta$. This is due to the pulse shape.

The probability density functions are outlined in Appen$\operatorname{dix}$ A. By substituting them in (4) and omitting the factors that are independent of $\theta$ and $\varepsilon$, the log-likelihood function can be rewritten as

$$
\Lambda(\theta, \varepsilon)=|\gamma(\theta)| \cos (2 \pi \varepsilon+\measuredangle \gamma(\theta))+\beta(\theta)
$$

where

$$
\begin{aligned}
& \gamma(\theta)=\sum_{k=\theta}^{\theta+L-1} h_{1}(k-\theta) r(k) r^{*}(k+N), \\
& \beta(\theta)=\sum_{k=-\infty}^{\infty} h_{2}(k-\theta)|r(k)|^{2},
\end{aligned}
$$

The two functions $\gamma(\theta)$ and $\beta(\theta)$ can be calculated by means of two filters with the statistics $r(k) r^{*}(k+N)$ and $|r(k)|^{2}$ as inputs. The filter coefficients are

$$
\begin{aligned}
& h_{1}(k)=\frac{2 g(k) g(k+N)}{g(k)^{2}+g(k+N)^{2}+\frac{1}{\mathrm{SNR}}}, \\
& h_{2}(k)= \begin{cases}-\frac{\operatorname{SNR} g(k+N)^{2}+1}{\operatorname{SNR}\left(g(k)^{2}+g(k+N)^{2}\right)+1} & k \in I \\
-\frac{\operatorname{SNR} g(k-N)^{2}+1}{\operatorname{SNR}\left(g(k)^{2}+g(k-N)^{2}\right)+1} & k \in I^{\prime} \\
-\frac{1}{\operatorname{SNR} g(k)^{2}+1} & k \notin I \cup I^{\prime}\end{cases}
\end{aligned}
$$

and depend on the expected power profile $g(k)^{2}$ and on the Signal-to-Noise Ratio, $\mathrm{SNR}=\sigma_{s}^{2} / \sigma_{n}^{2}$.

The filter $h_{2}$ is not implementable in the present form, because the filter coefficients in the interval $k \notin I \cup I^{\prime}$ are different from zero, although the pulse shape is finite-length. We can, however, make this filter implementable by adding the constant $\frac{1}{g_{\infty}^{2} \text { SNR }+1} \sum_{k=-\infty}^{\infty}|r(k)|^{2}$ to the log-likelihood function. The coefficients of the new filter $\tilde{h}_{2}$ become

$$
\tilde{h}_{2}(k)=\left\{\begin{array}{ll}
-\frac{\Delta_{\infty}+\mathrm{SNR}^{2} g(k+N)^{2} g_{\infty}^{2}-\Delta_{1}}{\Delta_{2} \Delta_{\infty}} & k \in I \\
-\frac{\Delta_{\infty}+\mathrm{SNR}^{2} g(k-N)^{2} g_{\infty}^{2}-\Delta_{1}}{\Delta_{3} \Delta_{\infty}} & k \in I^{\prime} \\
-\frac{\Delta_{\infty}-\Delta_{1}}{\Delta_{1} \Delta_{\infty}} & k \notin I \cup I^{\prime}
\end{array},\right.
$$

where

$$
\begin{aligned}
\Delta_{1} & =\operatorname{SNR} g(k)^{2}+1, \\
\Delta_{2} & =\operatorname{SNR} g(k)^{2}+\operatorname{SNR} g(k+N)^{2}+1, \\
\Delta_{3} & =\operatorname{SNR} g(k)^{2}+\operatorname{SNR} g(k-N)^{2}+1, \\
\Delta_{\infty} & =\operatorname{SNR} g_{\infty}^{2}+1,
\end{aligned}
$$

The joint ML-estimate of the OFDM of time offset $\theta$ and the frequency offset $\varepsilon$ is obtained by maximizing the loglikelihood function, 


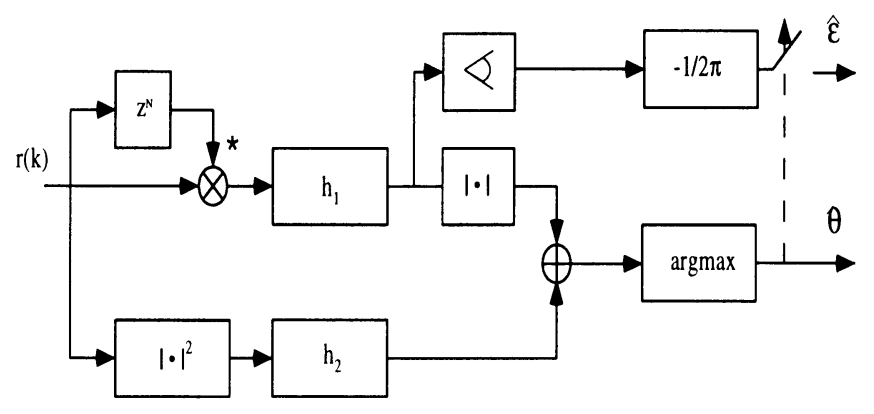

Figure 1: Structure of the estimator

$$
\begin{aligned}
& \widehat{\theta}_{\mathrm{ML}}=\arg \max _{\theta}\{|\gamma(\theta)|+\beta(\theta)\}, \\
& \widehat{\varepsilon}_{\mathrm{ML}}=\frac{-1}{2 \pi} \measuredangle \gamma\left(\widehat{\theta}_{\mathrm{ML}}\right) .
\end{aligned}
$$

The estimator can be realized with the structure of Figure 1. The filter $h_{1}$, used in (6), implements the first term of the argument of equation (15), and concerns the correlation introduced by the cyclic prefix. The second filter $h_{2}$, used in (7), can be viewed as a filter matched to the power profile and collects information supplied by the time-varying signal power.

\section{Power profile}

In this section we consider design criteria that affect the choice of the expected power profile $g(k)^{2}$. The pulse shape employed by the transmitter is of importance. We know that the received symbol, arriving at time $\theta$ with a frequency offset $\varepsilon$, has this pulse shape. An obvious choice is that $g(k)$ takes the values of this pulse shape on $k \in[0, N+L-1]$.

Outside the interval $k \in[0, N+L-1]$ the power profile $g(k)^{2}$ should ideally correspond to the actual received signal power. Neighbouring OFDM symbols, however, are likely to appear with time and frequency offsets, unknown to our estimator. More importantly, in a multiuser system, there may not be a neighbouring OFDM symbol. The profile $g(k)$ should be chosen to make the estimator robust against these uncertainties. In our simulations we have chosen to model the signal power of the last half of the preceding OFDM symbol and the first half of the following OFDM symbol. For samples further away, the profile is extended at a constant level. Thus, we allow our estimator to exploit information carried by the signal power close to our symbol, while disregarding information carried by samples further away. In order to account for the possible absence of a neighbouring symbol, we weigh the expected power profile outside the OFDM symbol of interest with an a priori probability that a neighbouring time slot is used. This probability may be interpreted as a system load.

In this model the jitter in time offset from neighboring symbols is not considered. This jitter can be included in our
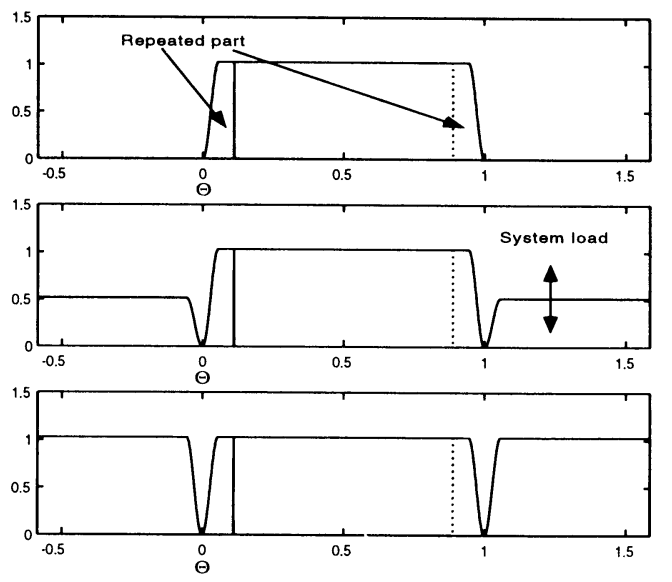

Figure 2: The function $g(k)$ for three different choices of system load, 0 (top), 0.5 (middle), and 1 (bottom).

model by averaging with an a priori probability function of the neighbouring symbols arrival time.

Figure 2 shows the profile $g(k)$ based on the Tukey pulse shape [12] for three choices of the system load. The uppermost part of Figure 2 shows $g(k)$ for a system load equal to zero. For a system load equal to one, $g(k)$ is shown in the lowermost part of Figure 2. Here, the adjacent time slots are used by other users. The middle picture of Figure 2 shows $g(k)$ for a system load equal to 0.5 , modelling uncertainty about the presence of neighbouring pulses.

\section{Performance Simulations}

We evaluate the estimator in a system where symbols are transmitted continuously (system load equals one), and do not overlap. The system has $N=1024$ tones and a cyclic prefix of $L=128$ samples. We consider two pulse shapes: the rectangular pulse shape as in [9] that carries no information in its power profile when the system load equals one, and the Tukey pulse shape where the outermost 32 samples on each side of the rectangular pulse shape are multiplied with a raised cosine. In the simulations we evaluate the estimator with the same pulse shape for which the estimator is derived.

Two channel models are used, the AWGN channel and the ETSI channel environment 'Vehicular B'. Figure 3 shows the simulated performance results for the AWGN channel. The curves show that the performance of the estimators is in the same order of magnitude in systems with and without pulse shaping. The loss in correlation due to the amplitude reduction in the redundant parts is partly compensated by the information in the time-varying power profile.

Figure 4 shows the performance of the estimator for the ETSI channel environment 'Vehicular B' [13]. The ETSI channel environment has an exponential decaying impulse 

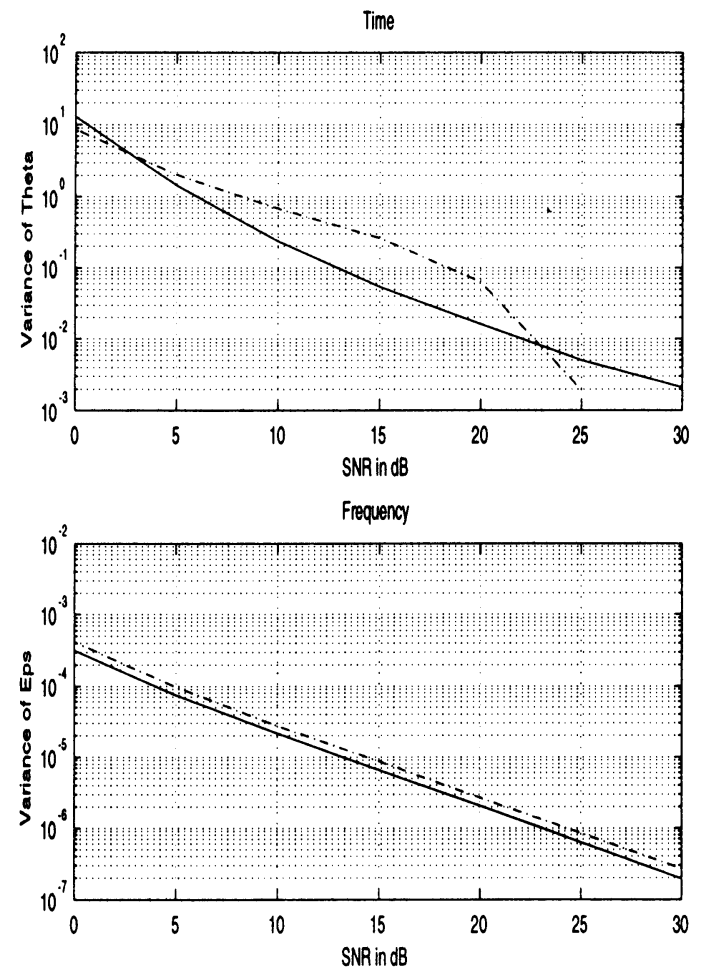

Figure 3: Performance of the time and frequency estimator for an AWGN channel, using the rectangular (solid) and the Tukey pulse shape (dashdotted).

response and fading characteristics according to the Jakes' model [14]. All other parameters are as above.

As expected, the estimator's performance decreases compared to the AWGN channel. The correlation properties of the received signal are changed due to the channel dispersion and this effect is not considered in our signal model. For the time offset estimation, the Tukey pulse shape may be more robust against the dispersion than the rectangular pulse shape. The rectangular window clearly has superior performance for the AWGN channel, but suffers from a high error floor for the dispersive channel. The increase in the error floor is due to two factors: the peak of the likelihood function is broadened by the dispersion and the data on the sides of the cyclic prefix mix with the repeated parts and act as strong additive noise.

For the AWGN channel the estimator performance may be improved by averaging the log-likelihood function over several OFDM symbols before detecting the maximum. For fading channel environments, performance improvement due to such averaging depends on the fading characteristics of the channel.
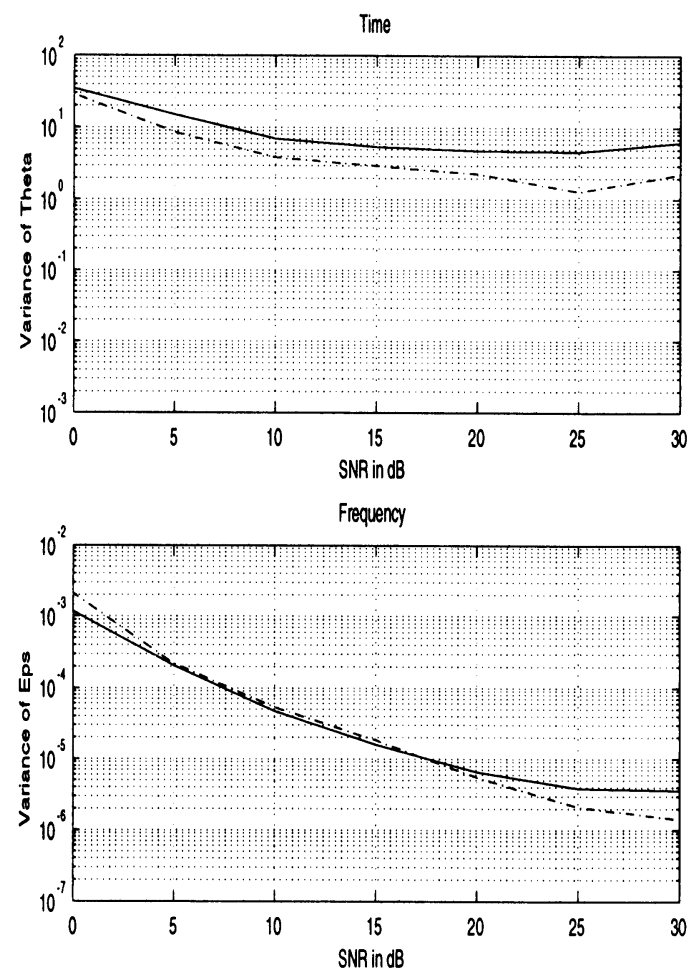

Figure 4: Performance of the time and frequency estimator for the ETSI channel 'Vehicular B', using the rectangular (solid) and the Tukey pulse shape (dasdotted).

\section{Conclusions}

In this paper the estimation of a time and frequency offset in a multi-user OFDM system is considered. The joint ML estimator is derived and an estimator structure is proposed. The estimator is based on the redundancy of the signal due to the cyclic prefix and on the power profile due to pulse shaping.

We draw two conclusions from this investigation. First, it is possible to extend the analytic techniques earlier employed in [9], deriving an implementable joint ML estimator, to systems with pulse shaping. Second, when introducing pulse shaping into an OFDM system it is possible to maintain the quality of the synchronization.

\section{Acknowledgment}

The authors wish to thank Sarah Kate Wilson for her constructive comments.

\section{A Probability density functions}

We define the two-variable, complex vector 


$$
\mathbf{x}=\left[\begin{array}{l}
x_{1} \\
x_{2}
\end{array}\right]=\left[\begin{array}{l}
r(k) \\
r(k+N)
\end{array}\right]
$$

The different probability density functions used in equation 4 can be expressed as

$$
\begin{gathered}
f(\mathbf{x})=k_{1} \exp \left(\frac{-1}{2} \mathbf{x}^{H} \mathbf{C}^{-1} \mathbf{x}\right) \\
f\left(x_{1}\right)=k_{2} \exp \left(\frac{-\left|x_{1}\right|^{2}}{\sigma_{s}^{2} g(k-\theta)^{2}+\sigma_{n}^{2}}\right), \\
f\left(x_{2}\right)=k_{3} \exp \left(\frac{-\left|x_{2}\right|^{2}}{\sigma_{s}^{2} g(k+N-\theta)^{2}+\sigma_{n}^{2}}\right),
\end{gathered}
$$

where $\sigma_{s}^{2} \equiv E\left\{|s(k)|^{2}\right\}$ and $\sigma_{n}^{2} \equiv E\left\{|n(k)|^{2}\right\}$. Here $k_{1}$, $k_{2}$ and $k_{3}$ are positive constants and $\mathbf{C}^{-1}$ is the inverse of the covariance matrix of the samples coupled through the cyclic prefix. In order to calculate this covariance matrix the correlation properties of the received signal are investigated. Thus, for $k \in I$, the different entries of the covariance matrix

$$
\mathbf{C}=\left[\begin{array}{ll}
c_{11} & c_{12} \\
c_{21} & c_{22}
\end{array}\right]
$$

are

$$
\begin{aligned}
c_{11} & =E\left\{x_{1} x_{1}^{*}\right\} \\
& =\sigma_{s}^{2} g(k-\theta)^{2}+\sigma_{n}^{2}, \\
c_{12} & =E\left\{x_{1} x_{2}^{*}\right\} \\
& =\sigma_{s}^{2} g(k-\theta) g(k+N-\theta) e^{-j 2 \pi \varepsilon}, \\
c_{21} & =E\left\{x_{1}^{*} x_{2}\right\} \\
& =\sigma_{s}^{2} g(k+N-\theta) g(k-\theta) e^{j 2 \pi \varepsilon}, \\
c_{22} & =E\left\{x_{2} x_{2}^{*}\right\} \\
& =\sigma_{s}^{2} g(k+N-\theta)^{2}+\sigma_{n}^{2} .
\end{aligned}
$$

Outside this interval the samples of the received signal are uncorrelated $\left(c_{12}=c_{21}=0\right)$.

\section{References}

[1] J. A. C. Bingham. Multicarrier modulation for data transmission: An idea whose time has come. IEEE Communications Magazine, 28(5):5-14, May 1990.

[2] L. Wei and C. Schlegel. Synchronization requirements for multi-user OFDM on satellite mobile and twopath Rayleigh fading channels. IEEE Trans. Comm., 43(2/3/4):887-895, Feb/Mar/Apr 1995.

[3] T. Pollet and M. Moeneclaey. Synchronizability of OFDM signals. In Proceedings of GLOBECOM'95, volume 3, pages 2054-2058. IEEE, November 1995.

[4] W. D. Warner and C. Leung. OFDM/FM frame synchronization for mobile radio data communication. IEEE Trans. Vehicular Tech., 42(3):302-313, August 1993.
[5] F. Daffara and O. Adami. A new frequency detector for orthogonal multicarrier transmission techniques. In Proc. VTC'95, volume 2, pages 804-809, Chicago, Illinois, USA, July 1995. IEEE.

[6] P. J. Tourtier, R. Monnier, and P. Lopez. Multicarrier modem for digital HDTV terrestrial broadcasting. Signal Processing: Image Communication, 5(5-6):379403, December 1993.

[7] P. H. Moose. A technique for orthogonal frequency division multiplexing frequency offset correction. IEEE Trans. Comm., 42(10):2908-2914, October 1994.

[8] J. J. van de Beek, M. Sandell, M. Isaksson, and P. O. $\mathrm{B}$ örjesson. Low-complex frame synchronization in OFDM systems. In Proc. of Intern. Conf. on Universal Personal Comm., pages 982-986. ICUPC '95, November 1995.

[9] J. J. van de Beek, M. Sandell, and P. O. Börjesson. ML Estimation of timing and frequency offset in OFDM systems. IEEE Trans. Signal Proc., in press.

[10] A. Vahlin and N. Holte. Optimal finite duration pulses for OFDM. IEEE Trans. Comm., 44(1):10-14, Jan 1996.

[11] R. Gross and D. Veeneman, Clipping Distortion in DMT ADSL Systems. Electron. Lett., Vol.29, no.24, pp.2080- 2081, November 1993.

[12] J. G. Proakis, D. G. Manolakis, Digital Signal Processing. Macmillan 2nd edition, 1992.

[13] D-ETR 50402 v.0.8.7. Selection procedures for the choice of radio transmission technologies of the Universal Mobile Telecommunication System (UMTS), May 1996.

[14] W. C. Jakes, Microwave Mobile Communications. Classic Reissue. IEEE Press, Piscataway, New Jersey, 1974. 